Herz 2020 · 45:325-326

https://doi.org/10.1007/s00059-020-04924-0

Published online: 24 April 2020

(c) Springer Medizin Verlag GmbH, ein Teil von Springer Nature 2020
Karl-Heinz Kuck ${ }^{1,2}$

'LANS Cardio, Hamburg, Germany

${ }^{2}$ Universitäres Herzzentrum, Universitätsklinikum Schleswig-Holstein - Campus Lübeck, Lübeck, Germany
Coronavirus disease 2019 (COVID19) is a global pandemic with almost 2 million infections confirmed worldwide and close to 120,000 deaths; the estimated mortality rate ranges from continent to continent between 3 and 9\% (as of April 15, 2020 [1]). The SARS$\mathrm{CoV}-2$ infection affects primarily adults, with fewer cases reported in children of 15 years or younger. The three primary symptoms of COVID-19 are fever, cough, and shortness of breath.

\section{Incidence of arrhythmias}

COVID-19 causes myocardial injury, with at least $17 \%$ of cases found to have an elevated troponin level and 23\% noted to have heart failure in a study of 191 inpatients from Wuhan, China [2]. Cases of fulminant myocarditis with cardiogenic shock have also been reported, with associated atrial and ventricular arrhythmias. In another study from China, patients with underlying cardiovascular disease (CVD) were more likely to exhibit elevation of troponin- $\mathrm{T}(\mathrm{TnT}$ ) levels ( $n=36,54.5 \%)$ compared with patients without CVD ( $n=16,13.2 \%)$. During hospitalization, patients with elevated TnT levels developed complications more frequently, including acute respiratory distress syndrome $(n=30,57.7 \%$ vs. $n=16,11.9 \%)$, malignant arrhythmias $(n=6,11.5 \%$ vs. $n=7,5.2 \%)$ including ventricular tachycardia/fibrillation, acute coagulopathy $(n=25,65.8 \%$ vs. $n=17,20.0 \%$ ), and acute kidney injury ( $n=14,36.8 \%$ vs. $n=4,4.7 \%$ ), compared with those with normal $\mathrm{TnT}$ levels [3].

Electrophysiologists play an important role in cardiovascular health, with more than $40 \%$ of symptoms in cardi- ology being arrhythmia-related. Besides myocardial infarction and heart failure, arrhythmias are generally one of the three major risks associated with viral infections, due to myocarditis, proinflammatory effects, and an increased sympathetic stimulation. In a report from Wuhan, China, $16.7 \%$ of hospitalized and $44.4 \%$ of intensive care unit (ICU) patients with COVID-19 had arrhythmias [4].

\section{Treatment}

Arrhythmias are a very common cardiac manifestation described in patients with COVID-19 infection. Symptoms can range from simple palpitations to lifethreating arrhythmias. Arrhythmias may be attributed to direct myocardial injury or occur secondary to metabolic derangement, hypoxia, neurohormonal changes, or inflammatory stress in the setting of acute viremia. However, a malignant arrhythmia in the setting of elevated cardiac markers should raise suspicion of underlying myocarditis. Given that hypoxia and electrolyte abnormalities that are common in the acute phase of severe illness can potentiate cardiac arrhythmias, the exact arrhythmic risk related to COVID-19 in patients with less severe illness or those who recover from the acute phase of the severe illness is currently unknown. Improved understanding of this is critical, primarily in guiding decisions on whether additional arrhythmia monitoring is needed (e.g., mobile cardiac telemetry) after discharge and whether an implantable cardioverter defibrillator (ICD) or wearable cardioverter defibrillator will be needed in those with impaired left ventricular function thought to be secondary to COVID-19.

\section{Hydroxychloroquine and QT prolongation}

It is unclear which medications may be beneficial for patients with COVID-19. Off-label use of some medications is currently being investigated. While no specific treatment is recommended, safety guidance for clinicians using hydroxychloroquine (HCQ) may be requested from electrophysiology (EP) providers. Hydroxychloroquine is known to block Kv11.1 (HERG) and can cause druginduced long-QT syndrome [5]. The clinical arrhythmic toxicity (syncope and torsade de pointes) is largely limited to chronic use (due to the agent's long half-life of 40 days), use of multiple concomitant QT-prolonging medications (e.g., azithromycin), metabolic derangements, renal failure, or in the setting of an acute overdose. To date, HCQ has been widely tolerated in most populations as an antimalarial medication and is safely used in rheumatoid arthritis and systemic lupus erythematosus populations without electrocardiogram (ECG) monitoring. Because the proposed HCQ therapy for COVID-19 is relatively short (e.g., 5-10 days), the risk of arrhythmic toxicity is likely quite low. There are specific precautions to be considered for select patients, however; patients with known congenital longQT syndrome and patients with severe renal insufficiency should have the dose reduced $(50 \%$ for $\mathrm{CrCl}<10 \mathrm{ml} / \mathrm{min})$, whereas patients on QT-prolonging drugs and electrolyte imbalances (e.g., hypokalemia, hypomagnesemia) should have these conditions corrected prior to use, with regular monitoring. 
It is also not clear from the literature which specific antiarrhythmic therapy should be used to prevent or suppress malignant ventricular arrhythmias in the setting of the COVID-19 infection. No specific therapy except external defibrillation/cardioversion has been described. The only medication that may be considered, as in other life-threatening situations, is amiodarone, but not in combination with chloroquine therapy.

\section{General recommendations for electrophysiologists}

Finally, the number of individuals in hospital rounding should be minimized and social distancing should be practiced. For patients with suspected or confirmed COVID-19 infection, the staff and the time spent in the room should also be limited. Many electrophysiology (EP) consultations may be completed without a face-to-face visit, by reviewing the chart and monitoring data. Non-urgent or non-emergent procedures should be postponed to a later date. Clinic visits and in-person cardiac implantable electronic device checks should be changed to tele-health and remote checks whenever feasible.

\section{Corresponding address}

Prof. Dr. med. Karl-Heinz Kuck LANS Cardio

Stephansplatz 5, 20354 Hamburg, Germany

kuckkh@aol.com

Conflict of interest. K.-H. Kuck declares that he has no competing interests.

\section{References}

1. World Health Organization (2020) Coronavirus disease (COVID-19) situation reports. https://www.who.int/emergencies/diseases/ novel-coronavirus-2019/situation-reports/. Accessed April 15, 2020

2. Zhou F et al (2020) Clinical course and risk factors for mortality of adult inpatients with COVID-19 in Wuhan, China: a retrospective cohort study. Lancet 395:1054-1062

3. Guo T et al (2020) Cardiovascular implications of fatal outcomes of patients with coronavirus disease 2019 (COVID-19). JAMA Cardiol. https:// doi.org/10.1001/jamacardio.2020.1017

4. Wang D et al (2020) Clinical characteristics of 138 hospitalized patients with 2019 novel coronavirusinfected pneumonia in Wuhan, China. JAMA. https://doi.org/10.1001/jama.2020.1585
5. Traebert $M$ et al (2004) Inhibition of hERG K+ currents by antimalarial drugs in stably transfected HEK293 cells. Eur J Pharmacol 484:41-48
Hier steht eine Anzeige.

Springer 